\title{
Corrigendum
}

\section{Quantity and species of fish consumed shape breast-milk fatty acid concentrations around Lake Victoria, Kenya}

\author{
Kathryn J Fiorella, Erin M Milner, Elizabeth Bukusi and Lia CH Fernald
}

First published online 6 March 2018

First published online - 27 November 2017

DOI: https://doi.org/10.1017/S1368980017003147

\section{Original text and correction:}

Kathryn J Fiorella ${ }^{(a 1)(a 2)}$, Erin M Milner ${ }^{(23)}$, Elizabeth Bukusi ${ }^{(a 4)}$ and Lia CH Fernald ${ }^{\text {(a3) }}$

(a1) Atkinson Center for a Sustainable Future, Cornell University, 340J Warren Hall, Ithaca, NY 14853, USA

(a2) Environmental Science, Policy \& Management, University of California-Berkeley, Berkeley, CA, USA

(a3) School of Public Health, University of California-Berkeley, Berkeley, CA, USA

(a4) Centre for Microbiology Research, Kenya Medical Research Institute, Kisumu, Kenya

\section{Correction:}

Kathryn J Fiorella (a1) (a2) (a3), Erin M Milner ${ }^{\text {(a4) }}$, Elizabeth Bukusi ${ }^{\text {(a5) }}$ and Lia CH Fernald ${ }^{\text {(a4) }}$

(a1) Master of Public Health Program, Population Medicine \& Diagnostic Sciences, College of Veterinary Medicine, Cornell University, Shurman Hall, Ithaca, NY 14853, USA

(a2) Atkinson Center for a Sustainable Future, Cornell University, 340J Warren Hall, Ithaca, NY 14853, USA

(a3) Environmental Science, Policy \& Management, University of California-Berkeley, Berkeley, CA, USA

(a4) School of Public Health, University of California-Berkeley, Berkeley, CA, USA

(a5) Centre for Microbiology Research, Kenya Medical Research Institute, Kisumu, Kenya 